Chinese Journal of Organic Chemistry

\title{
环状磷酸催化合成二吲哚甲烷衍生物
}

\author{
何 林*,a 王湘波 ${ }^{a}$ 杜广芬 ${ }^{a}$ 代 斌 ${ }^{a}$ 简腾跃 ${ }^{b}$ \\ ( ${ }^{a}$ 石河子大学化学化工学院 新疆兵团化工绿色过程重点实验室-省部共建国家重点实验室培育基地 \\ 石河子 832000) \\ $\left({ }^{b}\right.$ 中国科学院化学研究所 北京分子科学国家实验室 中国科学院分子识别与功能重点实验室 100190)
}

\begin{abstract}
摘要 在环状磷酸催化下, 通过吲哚和醛酮的亲电取代反应, 室温下合成了一系列二吲哚甲烷衍生物. 考察了催化剂 结构、用量和溶剂等因素对反应的影响, 确定了最优反应条件, 提出了可能的反应机理. 该方法具有操作简单、条件温 和、催化剂用量少、产率高及环境友好等优点.
\end{abstract}

关键词＼cjkstart吲哚; 醛; 酮; 环状磷酸; 二吲哚甲烷

\section{Synthesis of Bis(indolyl)methanes Catalyzed by Cyclic Phosphoric Acid}

\author{
He, Lin ${ }^{*, a} \quad$ Wang, Xiangbo ${ }^{a} \quad$ Du, Guangfen ${ }^{a} \quad$ Dai, Bin $^{a} \quad$ Jian, Tengyue $^{b}$ \\ ( ${ }^{a}$ Key Laboratory for Green Processing of Chemical Engineering of Xinjiang Bingtuan and School of Chemistry and Chemical \\ Engineering, Shihezi University, Shihezi 832000) \\ ( ${ }^{b}$ National Laboratory for Molecular Sciences, Chinese Academy of Sciences Key Laboratory of Molecular Recognition and \\ Function, Institute of Chemistry, Chinese Academy of Sciences, Beijing 100190)
}

\begin{abstract}
Cyclic phosphoric acid was found to be an efficient Brønsted acid catalyst for the electrophilic substitution reaction of indole with aldehydes or ketones in dichloromethane to afford the corresponding bis(indolyl)methanes in excellent yields at room temperature. The influence of catalyst structure, catalyst loading and reaction media was investigated, the optimal reaction conditions were obtained and a possible mechanism for the reaction was proposed. The simple procedure, mild reaction conditions, low catalyst loading, high yield and environmentally friendly organocatalyst provide a valuable approach for the preparation of bis(indolyl)methanes.
\end{abstract}

Keywords indole; aldehyde; ketone; cyclic phosphoric acid; bis(indolyl)methanes

二吲哚甲烷及其衍生物是陆地和海洋生物的一类 重要的生物活性代谢产物 ${ }^{[1]}$, 广泛存在各种天然产物中, 具有很好的生物活性和药物活性 ${ }^{[2]}$. 研究发现, 二吲哚 甲烷类物质具有抗癌、降血糖、抗病毒、抗菌和镇静等 作用 ${ }^{[3]}$, 在药品、农用化学品、材料科学等领域得到了 广泛的应用 ${ }^{[4]}$.

吲哚和醛、酮的亲电取代反应是制备二吲哚甲烷的 主要方法, 主要使用路易斯酸、质子酸、固载催化剂和 有机小分子催化剂等 ${ }^{[5]}$ 催化. 其中典型的催化剂有 $\mathrm{NH}_{4} \mathrm{Cl}^{[6]}$, 十二烷基苯磺酸 ${ }^{[7]}$, $(\mathrm{COOH})_{2} \cdot \mathrm{H}_{2} \mathrm{O} / \mathrm{CTAB}^{[8]}$, $\mathrm{B}(\mathrm{OH})_{3}{ }^{[9]}$ 等质子酸催化剂以及蒙脱土 $\mathrm{K} 10-\mathrm{ZnCl}_{2}{ }^{[10]}$,
$\mathrm{SSA}^{[11]}, \mathrm{I}_{2}{ }^{[12]}, \mathrm{Cp}_{2} \mathrm{Zr}\left(\mathrm{OSO}_{2} \mathrm{C}_{8} \mathrm{~F}_{17}\right)_{2} \bullet \mathrm{THF}^{[13]}$ 和 $\mathrm{Eu}\left(\mathrm{NTf}_{2}{ }^{[14]}\right.$, $\mathrm{Ln}(\mathrm{OTf})_{3}{ }^{[15]}, \quad \operatorname{In}(\mathrm{OTf})_{3}{ }^{[16]}, \quad \mathrm{InCl}_{3}{ }^{[17]}, \mathrm{CuBr}_{2}{ }^{[18]}, \quad \mathrm{RuCl}_{3}$ • $3 \mathrm{H}_{2} \mathrm{O}^{[19]}, \mathrm{ZrOCl}_{2}$ 等 ${ }^{[20]}$ 过渡金属催化剂. 其中部分方法存 在反应温度高、催化剂用量大、催化剂贵、产率低、后 处理复杂以及不可避免的金属污染等缺点, 因而限制了 这些方法的应用. 因此, 开发更高效和环境友好的催化 体系来合成二吲哚甲烷衍生物具有很重要的意义. 自 Terada 等 ${ }^{[21]}$ 引环状磷酸催化剂以来, 环状磷酸作为一 种新型有机酸已经在有机小分子催化反应中得到了广 泛的应用. 2009 年, You 等 ${ }^{[22]}$ 报道了用环状磷酸催化合 成不对称的芳基二吲哚甲烷的方法. 最近, 我们利用环

*E-mail: helin@shzu.edu.cn

Received December 6, 2012; revised January 27, 2013; published online Febuary 5, 2013.

Project supported by the Team Innovation Project of Shihezi University (No. 2011ZRKETD-0403), the Key Laboratory of Molecular Recognition and Function, Chinese Academy of Sciences (No. 2009LMRF002).

石河子大学团队创新(No. 2011ZRKETD-0403)、中国科学院分子识别与功能重点实验室 2009 年开放基金(No. 2009LMRF002)资助项目. 
状磷酸 “一锅法” 催化合成了四取代咪唑化合物 ${ }^{[23]}$ 和 2-取代苯并咪唑化合物 ${ }^{[24]}$, 取得了很好的效果. 在此基 础上, 我们进一步对环状磷酸的催化性能进行研究, 发 现环状磷酸能够高效催化吲哚与羰基化合物反应合成 二吲哚甲烷，本文将对此做一报道.

\section{1 结果与讨论}

\section{1 结构表征}

产物的结构经熔点、 ${ }^{1} \mathrm{H}$ NMR 和 IR 光谱证实. 以化 合物 3a 为例: 实测熔点为 $76 \sim 78{ }^{\circ} \mathrm{C}$, 文献报道为 76 ${ }^{\circ} \mathrm{C}$; 在红外光谱中, 在 $3402 \mathrm{~cm}^{-1}$ 处的吸收峰为 $\mathrm{NH}$ 的伸 缩振动特征吸收峰, 在 $3050 \mathrm{~cm}^{-1}$ 为次甲基不对称伸缩 振动特征吸收峰; 在 $1600 \sim 1450 \mathrm{~cm}^{-1}$ 为苯环 $\mathrm{C}=\mathrm{C}$ 骨 架伸缩振动特征吸收峰; 在 $1089 \mathrm{~cm}^{-1}$ 为苯环上取代氯 原子的特征吸收峰; 在 $736 \mathrm{~cm}^{-1}$ 为吲哚苯环邻位二取代 特征吸收峰; 核磁共振氢谱中, 在化学位移 $\delta 7.85$ 出现 吲哚杂环 $(\mathrm{N}-\mathrm{H})$ 中活波氢的单峰, 氢个数为 2 个; 在 $\delta$ 7.34 6.97 出现芳氢的一组双峰, 一组 $\mathrm{dt}$ 峰和四组 $\mathrm{m}$ 峰, 芳氢个数为 12 个; 在 $\delta 6.55$ 出现吲哚杂环 $(\mathrm{C}-\mathrm{H})$ 的一组 $\mathrm{dd}$ 峰, 2 个氢; 在 $\delta 5.82$ 出现次甲基 $(\mathrm{C}-\mathrm{H})$ 的单峰, 1 个 氢, 目标化合物与文献报道数据相符.

\section{2 反应条件的优化}

\subsection{1 催化剂对反应的影响}

在室温下, 以四氢呋喃作反应溶剂, 反应时间为 6 $\mathrm{h}$, 催化剂用量为 $10 \mathrm{~mol} \%$ 情况下, 考察了 5 种不同催化 剂对反应的影响(Eq. 1). 结果见表 1. 结果表明, 5 种不 同催化剂对反应都有催化活性, 其中对于氮原子上连有 供电子取代基的催化剂 $4 \mathbf{a}$ 的催化效率最高, 产率可达 到 98\%(表 1, Entry 1). 催化剂 4b 对该反应也有很好的催 化活性(表 1, Entry 2). 对于催化剂 4c, 催化产率为 75\%, 吲哚原料回收率为 $22 \%$ (20.4 mg, 表 1 , Entry 3). 对于氮 原子上连有强吸电子取代基的催化剂 $4 \mathbf{d}$, 催化产率只 有 71\%, 吲哚原料回收率为 $27 \%(25.0 \mathrm{mg}$, 表 1, Entry 4). 结果表明, 氮原子上不同取代基的催化剂催化活性 不同, 供电子取代基的催化活性高于强吸电子基. 基于 联菜酚合成的磷酸催化剂 $\mathbf{5}$, 催化产率仅为 $53 \%$, 吲哚 原料回收率为 43\% (40.4 mg, 表 1, Entry 5). 综合考虑催 化剂的成本和制备的难易程度及其催化活性，选用 $4 \mathbf{a}$ 作为合成二吲哚甲烷的催化剂.

\subsection{2 不同溶剂对反应的影响}

室温条件下, 以 $10 \mathrm{~mol} \%$ 4a 的催化剂用量, 考察了 乙醚、二氯甲烷、乙醇、甲醇、乙腈、1,4-二氧六环和 甲苯等不同溶剂对反应的影响, 结果见表 2. 可以看出, 溶剂对此反应影响不大, 但在二氯甲烷作溶剂时, 反应
时间短，产率为 98\%(表 2, Entry 3). 考虑到催化剂在溶 剂中的溶解性和催化活性, 本实验确定二氯甲烷为反应 溶剂.
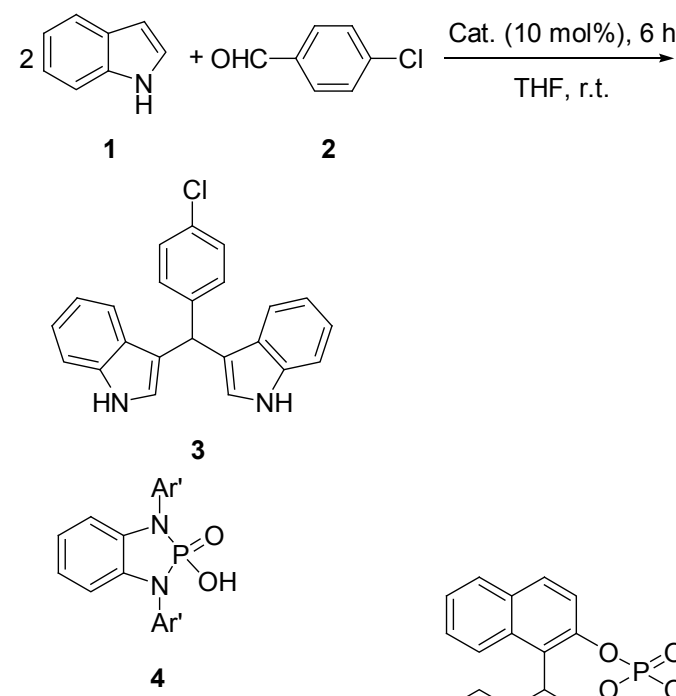

4a: $\mathrm{Ar}^{\prime}=4-\mathrm{CH}_{3} \mathrm{C}_{6} \mathrm{H}_{4} \mathrm{SO}_{2}$ 4b: $\mathrm{Ar}^{\prime}=4-\mathrm{H}_{3} \mathrm{COC}_{6} \mathrm{H}_{4} \mathrm{SO}_{2}$ 4c: $\mathrm{Ar}^{\prime}=\mathrm{C}_{6} \mathrm{H}_{5} \mathrm{SO}_{2}$

4d: $\mathrm{Ar}^{\prime}=4-\mathrm{NO}_{2} \mathrm{C}_{6} \mathrm{H}_{4} \mathrm{SO}_{2}$

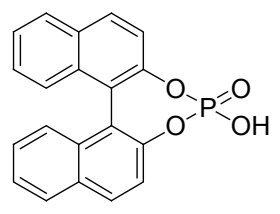

5

表 1 不同催化剂对反应产率的影响 ${ }^{a}$

Table 1 Effects of different catalysts on reaction yield

\begin{tabular}{ccl}
\hline Entry & Cat. & Yield $^{b} \%$ \\
\hline 1 & $\mathbf{4 a}$ & 98 \\
2 & $\mathbf{4 b}$ & 96 \\
3 & $\mathbf{4 c}$ & $75(22)^{c}$ \\
4 & $\mathbf{4 d}$ & $71(27)^{c}$ \\
5 & $\mathbf{5}$ & $53(43)^{c}$ \\
\hline
\end{tabular}

${ }^{a}$ Reaction conditions: indole $(0.8 \mathrm{mmol}), 4$-chlorobenzaldehyde $(0.4 \mathrm{mmol})$, catalyst $(10 \mathrm{~mol} \%)$, THF $(1 \mathrm{~mL})$, reaction time $6 \mathrm{~h}$, r.t. ${ }^{b}$ Isolated yields after silica gel column chromatography. ${ }^{c}$ The recovery of indole (\%).

表 2 不同溶剂对合成二吲哚甲烷的影响 ${ }^{a}$

Table 2 Solvents evaluation for bis(indolyl)methanes synthesis

\begin{tabular}{clcc}
\hline Entry & Solvent & Time $/ \mathrm{h}$ & Yield $^{b} \%$ \\
\hline 1 & $\mathrm{THF}$ & 6 & 98 \\
2 & $\mathrm{Et}_{2} \mathrm{O}$ & 6 & 96 \\
3 & $\mathrm{CH}_{2} \mathrm{Cl}_{2}$ & 3.5 & 98 \\
4 & $\mathrm{EtOH}$ & 5 & 94 \\
5 & $\mathrm{CH}_{3} \mathrm{CN}$ & 3.5 & 95 \\
6 & $1,4-\mathrm{Dioxane}$ & 5 & 94 \\
7 & $\mathrm{CH}_{3} \mathrm{OH}$ & 6 & 90 \\
8 & Toluene & 5 & 95 \\
\hline
\end{tabular}

${ }^{a}$ Reaction conditions: indole $(0.8 \mathrm{mmol})$, 4-chlorobenzaldehyde $(0.4 \mathrm{mmol})$, catalyst 4a $(10 \mathrm{~mol} \%)$, solvent $(1 \mathrm{~mL})$, r.t. ${ }^{b}$ Isolated yields after silica gel column chromatography.

\subsection{3 催化剂用量对反应的影响}

室温条件下, 以二氯甲烷为溶剂, 考察了催化剂 $\mathbf{4 a}$ 
用量对反应的影响, 结果见表 3 . 可以看出, 当催化剂 用量从 $10 \mathrm{~mol} \%$ 降低到 $1.5 \mathrm{~mol} \%$ 时，产率基本没有发生 变化(表 3, Entries 1 3), 进一步降低催化剂量到 1.0 mol\%, 产率仍可高达 97\%(表 3, Entry 4). 继续降低催化 剂量到 $0.5 \mathrm{~mol} \%$ 时, 产率降低到 $83 \%$ (表 3, Entry 5). 空 白实验表明，在不加催化剂情况下，即使延长反应时间 到 $12 \mathrm{~h}$, 产率只有 $1.5 \%$, 回收吲哚原料 $88.4 \mathrm{mg}$, 原料回 收率为 94\% (表 3, Entry 6). 所以, 本实验确定催化剂用

表 3 催化剂 $4 \mathbf{a}$ 用量对反应的影响 ${ }^{a}$

Table 3 Effects of amount of catalyst $4 \mathbf{a}$ on the reaction

\begin{tabular}{cccc}
\hline Entry & Cat. loading $/ \mathrm{mol} \%$ & Time/h & Yield $^{b} \%$ \\
\hline 1 & 10 & 3.5 & 98 \\
2 & 5.0 & 3.5 & 96 \\
3 & 1.5 & 3.5 & 95 \\
4 & 1.0 & 3.5 & 97 \\
5 & 0.5 & 3.5 & 83 \\
6 & - & 12 & $1.5(94)^{c}$ \\
\hline
\end{tabular}

${ }^{a}$ Reactions conditions: indole $(0.8 \mathrm{mmol})$, 4-chlorobenzaldehyde $(0.4 \mathrm{mmol})$, $\mathrm{CH}_{2} \mathrm{Cl}_{2}(1 \mathrm{~mL})$, r.t. ${ }^{b}$ Isolated yields after silica gel column chromatography.

${ }^{c}$ The recovery of indole (\%).
量为 $1.0 \mathrm{~mol} \%$ 对后续反应进行研究.

综合以上实验结果, 得到最优化的反应条件为：在 室温条件下，催化剂 $4 \mathrm{a}$ 用量为 $1.0 \mathrm{~mol} \%$, 溶剂为 $\mathrm{CH}_{2} \mathrm{Cl}_{2}$, TLC 跟踪监测反应进行程度, 并确定反应时间, 可以得到高产率的二吲哚甲烷衍生物.

\section{3 反应底物的普适性研究}

在最优化的反应条件下, 我们对该反应的普适性进 行了研究, 结果见表 4. 从实验结果可以看出, 各种取 代基的芳香醛都能顺利与吲哚进行反应，并可以得到较 高产率的目标化合物. 苯环上取代基的电子效应对反应 的影响较小, 不管是连有吸电子还是供电子的取代基都 能得到较高产率的化合物(表 4, Entries 1 5). 此外, 在 芳环不同位置进行取代，对反应影响不大，都能获得较 好的产率(表 4, Entries 1 8). 2,4-二氯苯甲醛和 2-菱醛 也是很好的底物, 与吲哚反应也能获得很好的产率(表 4, Entries 10, 11); 呋喃甲醛和胡椒醛与吲哚反应也能获 得高产率的目标化合物(表 4, Entries 12，13). 另外，我 们还考察了脂肪醛对反应的影响, 脂肪族醛也可以与吲

表 4 环状磷酸 $4 \mathbf{a}$ 催化合成二吲哚甲烷衍生物 ${ }^{a}$

Table 4 Synthesis of bis(indolyl)methanes catalyzed by cyclic phosphoric acid $4 \mathbf{a}$
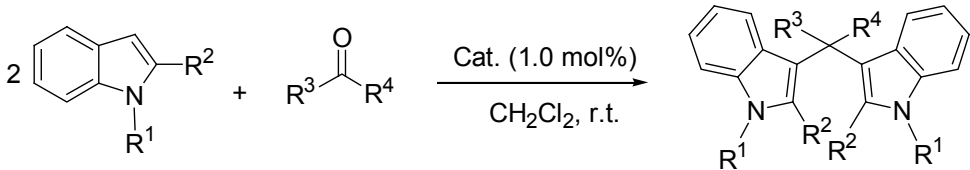

3

\begin{tabular}{|c|c|c|c|c|c|c|c|c|c|c|}
\hline Entry & $\mathrm{R}^{1}$ & $\mathrm{R}^{2}$ & $\mathrm{R}^{3}$ & $\mathrm{R}^{4}$ & Product & Time/h & Yield $^{b} / \%$ & m.p. $/{ }^{\circ} \mathrm{C}$ & Lit. $/{ }^{\circ} \mathrm{C}$ & Ref. \\
\hline 1 & $\mathrm{H}$ & $\mathrm{H}$ & $\mathrm{H}$ & 4-Cl- $\mathrm{C}_{6} \mathrm{H}_{4}$ & $3 a$ & 3.0 & 98 & $76 \sim 78$ & 76 & [25] \\
\hline 2 & $\mathrm{H}$ & $\mathrm{H}$ & $\mathrm{H}$ & $4-\mathrm{Br}-\mathrm{C}_{6} \mathrm{H}_{4}$ & $3 \mathbf{b}$ & 3.5 & 81 & $105 \sim 107$ & $110 \sim 112$ & [26] \\
\hline 3 & $\mathrm{H}$ & $\mathrm{H}$ & $\mathrm{H}$ & $4-\mathrm{NO}_{2}-\mathrm{C}_{6} \mathrm{H}_{4}$ & $3 c$ & 3.0 & 98 & $220 \sim 223$ & $220 \sim 222$ & [26] \\
\hline 4 & $\mathrm{H}$ & $\mathrm{H}$ & $\mathrm{H}$ & 4- $\mathrm{CH}_{3}-\mathrm{C}_{6} \mathrm{H}_{4}$ & 3d & 3.0 & 96 & $98 \sim 100$ & $96 \sim 98$ & [25] \\
\hline 5 & $\mathrm{H}$ & $\mathrm{H}$ & $\mathrm{H}$ & $4-\mathrm{MeO}-\mathrm{C}_{6} \mathrm{H}_{4}$ & $3 e$ & 4.0 & 84 & $190 \sim 193$ & $190 \sim 192$ & [25] \\
\hline 6 & $\mathrm{H}$ & $\mathrm{H}$ & $\mathrm{H}$ & $3-\mathrm{NO}_{2}-\mathrm{C}_{6} \mathrm{H}_{4}$ & $3 f$ & 3.0 & 97 & $86 \sim 88$ & $88 \sim 90$ & {$[25]$} \\
\hline 7 & $\mathrm{H}$ & $\mathrm{H}$ & $\mathrm{H}$ & $3-\mathrm{MeO}-\mathrm{C}_{6} \mathrm{H}_{4}$ & $3 g$ & 2.5 & 96 & $178 \sim 180$ & 189 & [19] \\
\hline 8 & $\mathrm{H}$ & $\mathrm{H}$ & $\mathrm{H}$ & $2-\mathrm{MeO}-\mathrm{C}_{6} \mathrm{H}_{4}$ & $3 \mathrm{~h}$ & 3.0 & 95 & $133 \sim 135$ & $134 \sim 136$ & [26] \\
\hline 9 & $\mathrm{H}$ & $\mathrm{H}$ & $\mathrm{H}$ & $\mathrm{C}_{6} \mathrm{H}_{5}$ & $3 \mathbf{i}$ & 2.5 & 97 & $86 \sim 88$ & $88 \sim 90$ & [25] \\
\hline 10 & $\mathrm{H}$ & $\mathrm{H}$ & $\mathrm{H}$ & $2,4-\mathrm{Cl}_{2}-\mathrm{C}_{6} \mathrm{H}_{3}$ & $3 \mathbf{j}$ & 2.5 & 97 & $102 \sim 105$ & $103 \sim 106$ & [26] \\
\hline 11 & $\mathrm{H}$ & $\mathrm{H}$ & $\mathrm{H}$ & 2-Naphtyl & $3 \mathbf{k}$ & 3.5 & 95 & $169 \sim 172$ & $235 \sim 236$ & {$[27]$} \\
\hline 12 & $\mathrm{H}$ & $\mathrm{H}$ & $\mathrm{H}$ & Furyl & 31 & 3.0 & 96 & $108 \sim 110$ & $113 \sim 115$ & [14] \\
\hline 13 & $\mathrm{H}$ & $\mathrm{H}$ & $\mathrm{H}$ & Piperonyl & $3 m$ & 6.0 & 91 & $95 \sim 98$ & $100 \sim 102$ & [28] \\
\hline 14 & $\mathrm{H}$ & $\mathrm{H}$ & $\mathrm{H}$ & $\mathrm{CH}_{3} \mathrm{CH}_{2} \mathrm{CH}_{2}$ & $3 n$ & 6.0 & $65(31)^{d}$ & $105 \sim 107$ & $106 \sim 108$ & [25] \\
\hline 15 & $\mathrm{H}$ & $\mathrm{H}$ & $\mathrm{H}$ & $\mathrm{C}_{6} \mathrm{H}_{5} \mathrm{CH}_{2} \mathrm{CH}_{2}$ & 30 & 4.0 & 83 & $80 \sim 82$ & $82 \sim 84$ & [29] \\
\hline 16 & $\mathrm{H}$ & $\mathrm{H}$ & $\mathrm{H}$ & Cyclohexyl & $3 p$ & 6.0 & 84 & $133 \sim 134$ & $136 \sim 137$ & [30] \\
\hline 17 & $\mathrm{CH}_{3}$ & $\mathrm{H}$ & $\mathrm{H}$ & $\mathrm{C}_{6} \mathrm{H}_{5}$ & $3 q$ & 4.0 & $73(16)^{d}$ & $190 \sim 193$ & $185 \sim 187$ & [19] \\
\hline 18 & $\mathrm{CH}_{3}$ & $\mathrm{H}$ & $\mathrm{H}$ & 4- $\mathrm{CH}_{3}-\mathrm{C}_{6} \mathrm{H}_{4}$ & $3 r$ & 5.5 & $73(11)^{d}$ & $197 \sim 200$ & $198 \sim 200$ & [31] \\
\hline 19 & $\mathrm{H}$ & $\mathrm{CH}_{3}$ & $\mathrm{H}$ & $\mathrm{C}_{6} \mathrm{H}_{5}$ & $3 s$ & 2.0 & 95 & $102 \sim 104$ & $247 \sim 248$ & {$[2]$} \\
\hline 20 & $\mathrm{H}$ & $\mathrm{CH}_{3}$ & $\mathrm{H}$ & $4-\mathrm{NO}_{2}-\mathrm{C}_{6} \mathrm{H}_{4}$ & $3 t$ & 1.5 & 97 & $232 \sim 234$ & $239 \sim 241$ & {$[2]$} \\
\hline 21 & $\mathrm{H}$ & $\mathrm{CH}_{3}$ & $\mathrm{H}$ & $4-\mathrm{CH}_{3}-\mathrm{C}_{6} \mathrm{H}_{4}$ & $3 u$ & 2.0 & 94 & $102 \sim 104$ & $106 \sim 108$ & [32] \\
\hline $22^{c}$ & $\mathrm{H}$ & $\mathrm{H}$ & $\mathrm{CH}_{3}$ & $\mathrm{C}_{6} \mathrm{H}_{5}$ & $3 v$ & 20 & $19(78)^{d}$ & $187 \sim 189$ & $189 \sim 190$ & [30] \\
\hline $23^{c}$ & $\mathrm{H}$ & $\mathrm{H}$ & $\mathrm{CH}_{3}$ & $\mathrm{CH}_{3}$ & $3 w$ & 20 & 82 & $158 \sim 160$ & $167 \sim 168$ & [26] \\
\hline
\end{tabular}

${ }^{a}$ Reaction conditions: indole $(0.8 \mathrm{mmol})$, aldehyde $(0.4 \mathrm{mmol})$, catalyst $4 \mathbf{a}(1 \mathrm{~mol} \%), \mathrm{CH}_{2} \mathrm{Cl}_{2}(1 \mathrm{~mL})$, r.t. ${ }^{b}$ Isolated yields after silica gel column chromatography. ${ }^{c}$ Indole $(0.8 \mathrm{mmol})$, ketone $(0.8 \mathrm{mmol})$, catalyst $4 \mathbf{a}(5 \mathrm{~mol} \%) .{ }^{d}$ The recovery of indole $(\%)$. 
哚进行反应, 但与芳香醛相比, 其产率明显降低(表 4, Entries $14 \sim 16$ ). 苯丙醛和环己基甲醛与吲哚反应可以 较高产率得到目标产物(表 4, Entries 15, 16). 但丁醛与 吲哚反应的活性最低, 仅得到产率为 $65 \%$ 的目标产物, 吲哚原料回收率为 31\% (29.7 mg, 表 4, Entry 14). 最后, 我们考察了吲哚环上不同取代基位置对反应的影响, 结 果表明, 取代基位置对反应的影响较大, 吲哚 $\mathrm{N}$ 上的 $\mathrm{H}$ 被甲基取代时, 活性降低, 相应的产率较少(表 4, Entries $17,18)$. 当 $N$-甲基吲哚与苯甲醛反应时得到产率为 $73 \%$ 的目标产物, $N$-甲基吲哚原料回收率为 16\% (16.4 mg, 表 4, Entry 17); $N$-甲基吲哚与对甲基苯甲醛反应时得到 产率为 $73 \%$ 的目标产物, $N$-甲基吲哚原料回收率为 $11 \%$ (11.7 mg, 表 4, Entry 18). 当吲哚 2 位上的 $\mathrm{H}$ 被甲基取 代时, 与没有被取代相当, 都能得到高产率的目标化合 物(表 4, Entries 19 21). 而当吲哚与酮反应时, 即使催 化剂用量增加到 $5 \mathrm{~mol} \%$, 酮当量加倍, 反应时间延长到 $20 \mathrm{~h}$, 也只能得到较低产率的产品(表 4, Entries 22, 23), 当吲哚与丙酮反应时, 可以得到产率为 $82 \%$ 的目标产 物. 但是, 当吲哚与苯丙酮反应时, 由于苯丙酮极低的 活性, 最终仅得到产率为 $19 \%$ 的目标产物, 吲哚原料回 收率为 78\% (73.0 mg, 表 4, Entry 23).

\section{4 反应机理}

在前人研究环状磷酸催化反应的基础上 ${ }^{[22]}$, 我们 提出了 Brønsted 酸催化机理(Scheme 1). 环状磷酸通过 氢键活化羰基氧, 然后吲哚与活化了的羰基碳进行反应 得到中间体 4; 脱去一分子水后得到中间体氮杂富烯盐 6, 中间体盐 6 与另一分子吲哚进一步反应, 并释放出氢 离子，最终得到二吲哚甲烷目标化合物 9.

\section{2 结论}

在环状磷酸催化下, 通过吲哚与醛或酮在室温下的 反应, 合成了一系列收率高的二吲哚甲烷衍生物. 该法
操作简便、产率高、后处理方便、催化剂用量少，催化 剂对空气稳定且环境友好, 这为该类化合物的合成提供 了一种简单、有效的绿色合成方法. 吲哚与脂肪醛和酮 的反应没有达到预期的效果，有待于进一步研究.

\section{3 实验部分}

\section{1 仪器与试剂}

熔点测定使用 X-5 显微熔点测定仪(控温型), 温度 未经校正; 核磁共振氢谱(400 MHz)采用美国 Varian 公 司 INOVA-400 MHz 型核磁共振仪测定, 用 $\mathrm{CDCl}_{3}$ 为溶 剂, TMS 为内标; 红外光谱用 Nicolet AVATAR-360 FT-IR 红外光谱仪 $(\mathrm{KBr}$ 压片)测定. 所有药品和试剂均 为分析纯, 苯甲醛、正丁醛和呋喃甲醛需重蒸, 其他试 剂未加处理直接使用.

\section{2 二吲哚甲烷化合物的合成方法}

将 $0.8 \mathrm{mmol}$ 吲哚、 $0.4 \mathrm{mmol}$ 醛或酮和 $1 \mathrm{~mL} \mathrm{CH}_{2} \mathrm{Cl}_{2}$ 加入 $25 \mathrm{~mL}$ 反应瓶中, 然后加入 $2.0 \mathrm{mg}$ 催化剂. 室温下 搅拌反应, TLC 跟踪监测反应进行程度, 直至原料消失 后停止反应, 粗产物通过柱层析分离纯化 $[V($ 石油 醚) $: V($ 乙酸乙酯 $)=10 ： 1 \sim 2 ： 1]$, 即可得到纯的目标 产物.

3,3'-二吲哚基-(4-氯苯基)甲烷(3a): 得 $140 \mathrm{mg}$ 白色 固体, 产率 98\%. m.p. $76 \sim 78{ }^{\circ} \mathrm{C}$ (lit. ${ }^{[25]} 76{ }^{\circ} \mathrm{C}$ ); ${ }^{1} \mathrm{H}$ NMR (400 MHz, $\left.\mathrm{CDCl}_{3}\right) \delta: 7.85$ (s, 2H), 7.34 (d, $J=8.0$ $\mathrm{Hz}, 2 \mathrm{H}), 7.30(\mathrm{dt}, J=8.2,0.8 \mathrm{~Hz}, 2 \mathrm{H}), 7.25 \sim 7.22(\mathrm{~m}$, $2 \mathrm{H}), 7.21 \sim 7.18(\mathrm{~m}, 2 \mathrm{H}), 7.17 \sim 7.13(\mathrm{~m}, 2 \mathrm{H}), 7.02 \sim 6.97$ $(\mathrm{m}, 2 \mathrm{H}), 6.55$ (dd, $J=2.4,0.9 \mathrm{~Hz}, 2 \mathrm{H}), 5.82$ (s, 1H); IR (KBr) v: 3402, 3050, 1600, 1490, 1457, 1409, 1331, 1089, $1014,736 \mathrm{~cm}^{-1}$.

3,3'-二吲哚基-(4-溴苯基)甲烷(3b): 得 $130 \mathrm{mg}$ 白色 固体，产率 $81 \%$. m.p. $105 \sim 107{ }^{\circ} \mathrm{C}$ (lit. ${ }^{[26]} 110 \sim 112$

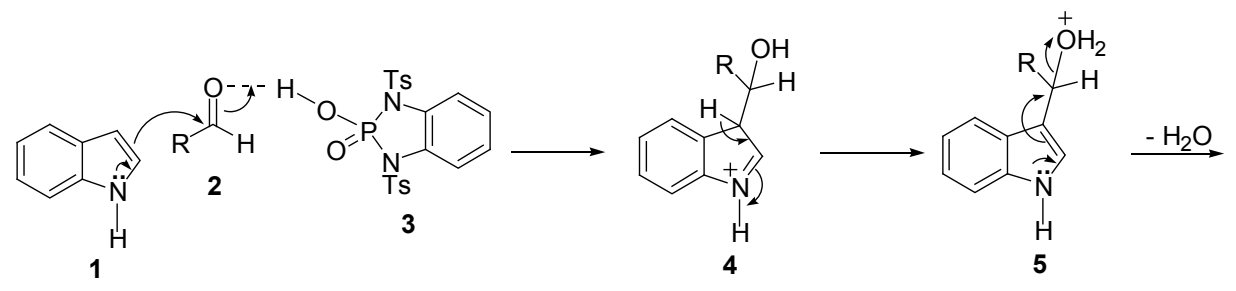

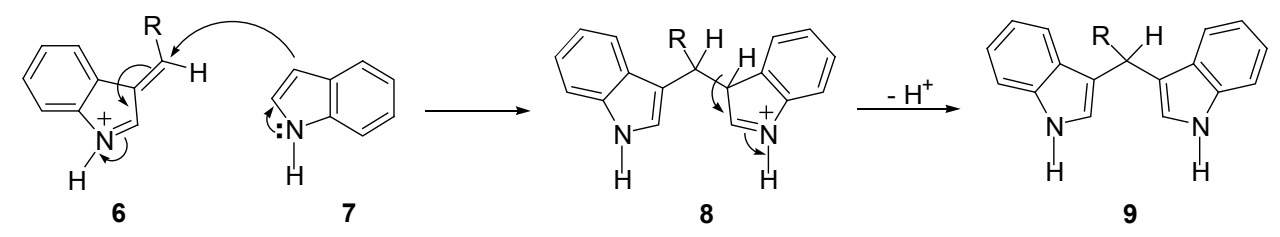

Scheme 1 
$\left.{ }^{\circ} \mathrm{C}\right) ;{ }^{1} \mathrm{H}$ NMR $\left(400 \mathrm{MHz}, \mathrm{CDCl}_{3}\right) \delta: 7.92(\mathrm{~s}, 2 \mathrm{H}), 7.38 \sim$ $7.34(\mathrm{~m}, 4 \mathrm{H}), 7.34 \sim 7.32(\mathrm{~m}, 2 \mathrm{H}), 7.20 \sim 7.17(\mathrm{~m}, 2 \mathrm{H})$, $7.17 \sim 7.13(\mathrm{~m}, 2 \mathrm{H}), 7.02 \sim 6.97(\mathrm{~m}, 2 \mathrm{H}), 6.61(\mathrm{dd}, J=2.4$, $1.0 \mathrm{~Hz}, 2 \mathrm{H}), 5.82$ (s, 1H); IR (KBr) v: 3410, 3057, 1619, $1481,1453,1006,745 \mathrm{~cm}^{-1}$.

3,3'-二吲哚基-(4-硝基苯基)甲烷(3c): 得 $144 \mathrm{mg}$ 棕 色固体, 产率 98\%. m.p. $220 \sim 223{ }^{\circ} \mathrm{C}$ (lit. ${ }^{[26]} 220 \sim 222$ $\left.{ }^{\circ} \mathrm{C}\right) ;{ }^{1} \mathrm{H}$ NMR $\left(400 \mathrm{MHz}, \mathrm{CDCl}_{3}\right) \delta: 8.16 \sim 8.13(\mathrm{~m}, 2 \mathrm{H})$, $8.02(\mathrm{~s}, 2 \mathrm{H}), 7.53 \sim 7.50(\mathrm{~m}, 2 \mathrm{H}), 7.39(\mathrm{dt}, J=8.2,0.8 \mathrm{~Hz}$, $2 \mathrm{H}), 7.36 \sim 7.33(\mathrm{~m}, 2 \mathrm{H}), 7.23 \sim 7.18(\mathrm{~m}, 2 \mathrm{H}), 7.06 \sim 7.01$ (m, 2H), 6.69 (dd, $J=2.5,1.0 \mathrm{~Hz}, 2 \mathrm{H}), 6.00$ (s, 1H); IR (KBr) v: 3453, 3417, 3382, 1596, 1502, 1457, 1414, 1343, $1097,1010,808,745 \mathrm{~cm}^{-1}$.

3,3'-二吲哚基-(4-甲基苯基)甲烷(3d): 得 $129 \mathrm{mg}$ 粉 红色固体, 产率 96\%. m.p. 98 $\sim 100{ }^{\circ} \mathrm{C}$ (lit. ${ }^{[25]}$ 96 98 $\left.{ }^{\circ} \mathrm{C}\right) ;{ }^{1} \mathrm{H}$ NMR $\left(400 \mathrm{MHz}, \mathrm{CDCl}_{3}\right) \delta: 7.81(\mathrm{~s}, 2 \mathrm{H}), 7.38 \sim$ $7.35(\mathrm{~m}, 2 \mathrm{H}), 7.30(\mathrm{dt}, J=8.2,0.9 \mathrm{~Hz}, 2 \mathrm{H}), 7.21 \sim 7.18(\mathrm{~m}$, $2 \mathrm{H}), 7.15 \sim 7.11(\mathrm{~m}, 2 \mathrm{H}), 7.07 \sim 7.03(\mathrm{~m}, 2 \mathrm{H}), 7.00 \sim 6.95$ (m, 2H), 6.59 (dd, $J=2.4,1.0 \mathrm{~Hz}, 2 \mathrm{H}), 5.82(\mathrm{~s}, 1 \mathrm{H}), 2.29$ (s, 3H); IR (KBr) v: 3406, 3053, 2927, 1612, 1513, 1453, 1409, 1335, 1219, 1085, 1002, 780, $745 \mathrm{~cm}^{-1}$.

3,3'-二吲哚基-(4-甲氧基苯基)甲烷(3e): 得 $118 \mathrm{mg}$ 棕色固体, 产率 84\%. m.p. 190 193 ${ }^{\circ} \mathrm{C}$ (lit. ${ }^{[25]} 190 \sim$ $\left.192{ }^{\circ} \mathrm{C}\right) ;{ }^{1} \mathrm{H}$ NMR $\left(400 \mathrm{MHz}, \mathrm{CDCl}_{3}\right) \delta: 7.89$ (s, 2H), $7.40 \sim 7.37(\mathrm{~m}, 2 \mathrm{H}), 7.30(\mathrm{dt}, J=8.1,0.9 \mathrm{~Hz}, 2 \mathrm{H}), 7.26 \sim$ $7.25(\mathrm{~m}, 1 \mathrm{H}), 7.24 \sim 7.23(\mathrm{~m}, 1 \mathrm{H}), 7.18 \sim 7.13(\mathrm{~m}, 2 \mathrm{H})$, $7.01 \sim 6.97(\mathrm{~m}, 2 \mathrm{H}), 6.83 \sim 6.79(\mathrm{~m}, 2 \mathrm{H}), 6.64(\mathrm{dd}, J=2.4$, $1.0 \mathrm{~Hz}, 2 \mathrm{H}), 5.83$ (s, 1H), 3.77 (s, 3H); IR (KBr) v: 3398, 3057, 1606, 1517, 1450, 1247, 1021, $737 \mathrm{~cm}^{-1}$.

3,3'-二吲哚基-(3-硝基苯基)甲烷(3f): 得 $142 \mathrm{mg}$ 浅 黄色固体, 产率 $97 \%$. m.p. $86 \sim 88{ }^{\circ} \mathrm{C}$ (lit. ${ }^{[25]} 88 \sim 90$ $\left.{ }^{\circ} \mathrm{C}\right) ;{ }^{1} \mathrm{H}$ NMR (400 MHz, $\left.\mathrm{CDCl}_{3}\right) \delta: 8.19(\mathrm{t}, J=2.0 \mathrm{~Hz}$, $1 \mathrm{H}$ ), 8.06 (ddd, $J=3.3,2.3,1.0 \mathrm{~Hz}, 1 \mathrm{H}$ ), 7.96 (s, 2H), 7.66 (d, $J=7.7 \mathrm{~Hz}, 1 \mathrm{H}), 7.41(\mathrm{t}, J=8.0 \mathrm{~Hz}, 1 \mathrm{H}), 7.36 \sim 7.32$ $(\mathrm{m}, 4 \mathrm{H}), 7.20 \sim 7.15(\mathrm{~m}, 2 \mathrm{H}), 7.04 \sim 6.99(\mathrm{~m}, 2 \mathrm{H}), 6.61(\mathrm{~d}$, $J=2.4 \mathrm{~Hz}, 2 \mathrm{H}), 5.97$ (s, 1H); IR (KBr) v: 3406, 3061, $1612,1520,1457,1417,1354,1101,748 \mathrm{~cm}^{-1}$.

3,3'-二吲哚基-(3-甲氧基苯基)甲烷(3g): 得 $135 \mathrm{mg}$ 棕色固体, 产率 96\%. m.p. $178 \sim 180{ }^{\circ} \mathrm{C}$ (lit. ${ }^{[19]} 189{ }^{\circ} \mathrm{C}$ ); ${ }^{1} \mathrm{H}$ NMR (400 MHz, $\left.\mathrm{CDCl}_{3}\right) \delta: 7.90$ (s, 2H), 7.41 7.40 $(\mathrm{m}, 1 \mathrm{H}), 7.39 \sim 7.38(\mathrm{~m}, 1 \mathrm{H}), 7.34(\mathrm{dt}, J=8.2,0.9 \mathrm{~Hz}$, $2 \mathrm{H}), 7.21 \sim 7.18(\mathrm{~m}, 1 \mathrm{H}), 7.18 \sim 7.13(\mathrm{~m}, 2 \mathrm{H}), 7.02 \sim 6.98$ $(\mathrm{m}, 2 \mathrm{H}), 6.96 \sim 6.90(\mathrm{~m}, 2 \mathrm{H}), 6.75$ (ddd, $J=3.6,2.6,1.0$ $\mathrm{Hz}, 1 \mathrm{H}), 6.66$ (dd, $J=2.4,1.0 \mathrm{~Hz}, 2 \mathrm{H}), 5.85$ (s, 1H), 3.73 (s, 3H); IR (KBr) v: 3402, 3049, 1612, 1584, 1485, 1461,
$1330,1255,1210,1145,1089,1049,780,744 \mathrm{~cm}^{-1}$

3,3'-二吲哚基-(2-甲氧基苯基)甲烷(3h): 得 $134 \mathrm{mg}$ 红色固体, 产率 95\%. m.p. $133 \sim 135{ }^{\circ} \mathrm{C}$ (lit. ${ }^{[26]} 134 \sim$ $\left.136{ }^{\circ} \mathrm{C}\right) ;{ }^{1} \mathrm{H}$ NMR $\left(400 \mathrm{MHz}, \mathrm{CDCl}_{3}\right) \delta: 7.84(\mathrm{~s}, 2 \mathrm{H})$, $7.41 \sim 7.38(\mathrm{~m}, 2 \mathrm{H}), 7.30(\mathrm{dt}, J=8.1,0.8 \mathrm{~Hz}, 2 \mathrm{H}), 7.20 \sim$ $7.14(\mathrm{~m}, 2 \mathrm{H}), 7.13 \sim 7.10(\mathrm{~m}, 2 \mathrm{H}), 7.00 \sim 6.95(\mathrm{~m}, 2 \mathrm{H})$, $6.92(\mathrm{dd}, J=8.2,1.0 \mathrm{~Hz}, 1 \mathrm{H}), 6.79$ (td, $J=7.4,1.0 \mathrm{~Hz}$, $1 \mathrm{H}), 6.60$ (dd, $J=2.3,0.9 \mathrm{~Hz}, 2 \mathrm{H}), 6.34$ (s, 1H), 3.80 (s, 3H); IR (KBr) v: 3406, 3061, 1738, 1592, 1493, 1461, 1338, 1237, 1101, 1042, $748 \mathrm{~cm}^{-1}$.

3,3'-二吲哚基苯甲烷(3i): 得 $125 \mathrm{mg}$ 白色固体, 产 率 97\%. m.p. $86 \sim 88{ }^{\circ} \mathrm{C}$ (lit. ${ }^{[25]} 88 \sim 90{ }^{\circ} \mathrm{C}$ ); ${ }^{1} \mathrm{H}$ NMR $\left(400 \mathrm{MHz}, \mathrm{CDCl}_{3}\right) \delta: 7.81(\mathrm{~s}, 2 \mathrm{H}), 7.39 \sim 7.36(\mathrm{~m}, 2 \mathrm{H})$, $7.34 \sim 7.32(\mathrm{~m}, 1 \mathrm{H}), 7.32 \sim 7.30(\mathrm{~m}, 2 \mathrm{H}), 7.29(\mathrm{t}, J=0.9$ $\mathrm{Hz}, 1 \mathrm{H}), 7.27 \sim 7.23(\mathrm{~m}, 2 \mathrm{H}), 7.20(\mathrm{dt}, J=7.2,1.6 \mathrm{~Hz}$, $1 \mathrm{H}), 7.17 \sim 7.12(\mathrm{~m}, 2 \mathrm{H}), 7.01 \sim 6.96(\mathrm{~m}, 2 \mathrm{H}), 6.57(\mathrm{dd}$, $J=2.4,1.0 \mathrm{~Hz}, 2 \mathrm{H}), 5.86(\mathrm{~s}, 1 \mathrm{H})$; IR (KBr) $v: 3406,3057$, $1485,1457,1414,1339,1212,1085,1014,745 \mathrm{~cm}^{-1}$.

$3,3^{\prime}$-二吲哚基-(2,4-二氯苯基)甲烷(3j): 得 $151 \mathrm{mg}$ 棕色固体, 产率 97\%. m.p. 102 105 ${ }^{\circ} \mathrm{C}$ (lit. ${ }^{[26]} 103 \sim$ $\left.106{ }^{\circ} \mathrm{C}\right) ;{ }^{1} \mathrm{H}$ NMR $\left(400 \mathrm{MHz}, \mathrm{CDCl}_{3}\right) \delta: 7.83$ (s, 2H), 7.43 (d, $J=2.1 \mathrm{~Hz}, 1 \mathrm{H}), 7.36(\mathrm{~s}, 1 \mathrm{H}), 7.35(\mathrm{~s}, 1 \mathrm{H}), 7.33(\mathrm{~s}, 1 \mathrm{H})$, $7.31(\mathrm{~s}, 1 \mathrm{H}), 7.19 \sim 7.14(\mathrm{~m}, 2 \mathrm{H}), 7.13 \sim 7.10(\mathrm{~m}, 1 \mathrm{H})$, $7.07 \sim 6.99(\mathrm{~m}, 3 \mathrm{H}), 6.55(\mathrm{dd}, J=2.4,0.9 \mathrm{~Hz}, 2 \mathrm{H}), 6.25(\mathrm{~s}$, 1H); IR (KBr) v: 3410, 3057, 1584, 1457, 1414, 1334, 1244, 1093, 863, 796, $745 \mathrm{~cm}^{-1}$

3,3'二二吲哚基-(2-萗基)甲烷(3k): 得 $141 \mathrm{mg}$ 浅黄色 固体, 产率 $95 \%$. m.p. $169 \sim 172{ }^{\circ} \mathrm{C}$ (lit. ${ }^{[27]} 235 \sim 236$ $\left.{ }^{\circ} \mathrm{C}\right) ;{ }^{1} \mathrm{H}$ NMR (400 MHz, $\left.\mathrm{CDCl}_{3}\right) \delta: 7.88(\mathrm{~s}, 2 \mathrm{H}), 7.81 \sim$ $7.77(\mathrm{~m}, 1 \mathrm{H}), 7.76 \sim 7.72(\mathrm{~m}, 2 \mathrm{H}), 7.71 \sim 7.67(\mathrm{~m}, 1 \mathrm{H})$, $7.50(\mathrm{dd}, J=8.5,1.6 \mathrm{~Hz}, 1 \mathrm{H}), 7.42 \sim 7.38(\mathrm{~m}, 4 \mathrm{H}), 7.34(\mathrm{~s}$, $1 \mathrm{H}), 7.32(\mathrm{~s}, 1 \mathrm{H}), 7.17 \sim 7.12(\mathrm{~m}, 2 \mathrm{H}), 7.00 \sim 6.95(\mathrm{~m}$, 2H), 6.64 (s, 2H), 6.04 (s, 1H); IR (KBr) v: 3414, 3050, 2836, 1604, 1461, 1418, 1343, 1089, 807, $745 \mathrm{~cm}^{-1}$.

3,3'-二吲哚基-(2-呋喃基)甲烷(31): 得 $120 \mathrm{mg}$ 棕色 固体, 产率 96\%. m.p. $108 \sim 110{ }^{\circ} \mathrm{C}$ (lit. ${ }^{[14]} 113 \sim 115$ $\left.{ }^{\circ} \mathrm{C}\right) ;{ }^{1} \mathrm{H}$ NMR (400 MHz, $\left.\mathrm{CDCl}_{3}\right) \delta: 7.88(\mathrm{~s}, 2 \mathrm{H}), 7.48 \sim$ $7.47(\mathrm{~m}, 1 \mathrm{H}), 7.46 \sim 7.44(\mathrm{~m}, 1 \mathrm{H}), 7.34(\mathrm{dd}, J=1.8,0.9$ $\mathrm{Hz}, 1 \mathrm{H}), 7.31(\mathrm{dt}, J=8.2,0.9 \mathrm{~Hz}, 2 \mathrm{H}), 7.18 \sim 7.13(\mathrm{~m}$, $2 \mathrm{H}), 7.05 \sim 7.00(\mathrm{~m}, 2 \mathrm{H}), 6.82(\mathrm{dd}, J=2.4,0.8 \mathrm{~Hz}, 2 \mathrm{H})$, $6.28(\mathrm{dd}, J=3.0,1.9 \mathrm{~Hz}, 1 \mathrm{H}), 6.04(\mathrm{dt}, J=3.2,0.8 \mathrm{~Hz}$, 1H), $5.92(\mathrm{~d}, J=0.7 \mathrm{~Hz}, 1 \mathrm{H})$; IR $(\mathrm{KBr}) v: 3406,3046$, $1505,1457,1335,1097,1014,784,745 \mathrm{~cm}^{-1}$.

3,3'-二吲哚基-(3,4-环亚甲基苯基)甲烷(3m): 得 133 mg 棕色固体, 产率 91\%. m.p. 95 98 ${ }^{\circ} \mathrm{C}$ (lit. ${ }^{[28]} 100 \sim$ 
$\left.102{ }^{\circ} \mathrm{C}\right) ;{ }^{1} \mathrm{H}$ NMR $\left(400 \mathrm{MHz}, \mathrm{CDCl}_{3}\right) \delta: 7.85$ (s, 2H), $7.41 \sim 7.39(\mathrm{~m}, 1 \mathrm{H}), 7.39 \sim 7.37(\mathrm{~m}, 1 \mathrm{H}), 7.34(\mathrm{dt}, J=8.2$, $0.8 \mathrm{~Hz}, 2 \mathrm{H}), 7.18 \sim 7.13(\mathrm{~m}, 2 \mathrm{H}), 7.03 \sim 6.98(\mathrm{~m}, 2 \mathrm{H})$, $6.83 \sim 6.80(\mathrm{~m}, 2 \mathrm{H}), 6.73 \sim 6.70(\mathrm{~m}, 1 \mathrm{H}), 6.62(\mathrm{dd}, J=2.4$, $1.0 \mathrm{~Hz}, 2 \mathrm{H}), 5.88$ (s, 2H), 5.79 (s, 1H); IR (KBr) v: 3406, 3054, 1501, 1485, 1442, 1239, 1093, 1038, 931, 788, 745 $\mathrm{cm}^{-1}$.

3,3'-二吲哚基丁烷(3n): 得 $75 \mathrm{mg}$ 白色固体, 产率 $65 \%$. m.p. $105 \sim 107{ }^{\circ} \mathrm{C}$ (lit. ${ }^{[25]} 106 \sim 108{ }^{\circ} \mathrm{C}$ ); ${ }^{1} \mathrm{H}$ NMR $\left(400 \mathrm{MHz}, \mathrm{CDCl}_{3}\right) \delta: 7.87(\mathrm{~s}, 2 \mathrm{H}), 7.60 \sim 7.59(\mathrm{~m}, 1 \mathrm{H})$, $7.58 \sim 7.57(\mathrm{~m}, 1 \mathrm{H}), 7.26(\mathrm{dt}, J=8.1,0.9 \mathrm{~Hz}, 2 \mathrm{H}), 7.14 \sim$ $7.09(\mathrm{~m}, 2 \mathrm{H}), 7.04 \sim 6.98(\mathrm{~m}, 2 \mathrm{H}), 6.89(\mathrm{~d}, J=1.8 \mathrm{~Hz}$, $2 \mathrm{H}), 4.46(\mathrm{t}, J=7.4 \mathrm{~Hz}, 1 \mathrm{H}), 2.20 \sim 2.15(\mathrm{~m}, 2 \mathrm{H}), 1.44 \sim$ 1.37 (m, 2H), 0.93 (t, $J=7.3 \mathrm{~Hz}, 3 \mathrm{H})$; IR (KBr) $v: 3418$, 3057, 2951, 2927, 1537, 1457, 1422, 1339, 1212, 1101, $1006,741 \mathrm{~cm}^{-1}$.

3,3'-二吲哚基苯丙烷(3o): 得 $116 \mathrm{mg}$ 棕色固体, 产 率 $83 \%$. m.p. $80 \sim 82{ }^{\circ} \mathrm{C}$ (lit. $\left.{ }^{[29]} 82 \sim 84{ }^{\circ} \mathrm{C}\right)$; ${ }^{1} \mathrm{H}$ NMR $\left(400 \mathrm{MHz}, \mathrm{CDCl}_{3}\right) \delta: 7.85(\mathrm{~s}, 2 \mathrm{H}), 7.53(\mathrm{~d}, J=7.9 \mathrm{~Hz}$, $2 \mathrm{H}), 7.29(\mathrm{~d}, J=8.2 \mathrm{~Hz}, 2 \mathrm{H}), 7.27 \sim 7.23(\mathrm{~m}, 2 \mathrm{H}), 7.17 \sim$ $7.14(\mathrm{~m}, 3 \mathrm{H}), 7.07 \sim 7.03(\mathrm{~m}, 2 \mathrm{H}), 7.00 \sim 6.95(\mathrm{~m}, 2 \mathrm{H})$, $6.59(\mathrm{dd}, J=2.4,1.0 \mathrm{~Hz}, 2 \mathrm{H}), 4.49(\mathrm{t}, J=7.4 \mathrm{~Hz}, 1 \mathrm{H})$, $2.73 \sim 2.65$ (m, 2H), 2.54 (t, $J=7.9 \mathrm{~Hz}, 2 \mathrm{H})$; IR (KBr) $v$ : 3422, 3061, 3022, 2927, 1454, 1418, 1339, 1085, 1009, $750,701 \mathrm{~cm}^{-1}$.

3,3'-二吲哚基环己基甲烷(3p): 得 $110 \mathrm{mg}$ 棕色固体, 产率 84\%. m.p. $133 \sim 134{ }^{\circ} \mathrm{C}$ (lit. ${ }^{[30]} 136 \sim 137{ }^{\circ} \mathrm{C}$ ); ${ }^{1} \mathrm{H}$ NMR (400 MHz, $\left.\mathrm{CDCl}_{3}\right) \delta: 7.46(\mathrm{~s}, 2 \mathrm{H}), 7.18 \sim 7.00(\mathrm{~m}$, $8 \mathrm{H}), 6.73(\mathrm{~d}, J=2.2 \mathrm{~Hz}, 2 \mathrm{H}), 4.20 \sim 4.14(\mathrm{~m}, 1 \mathrm{H}), 2.20 \sim$ $2.10(\mathrm{~m}, 1 \mathrm{H}), 1.77 \sim 1.54(\mathrm{~m}, 10 \mathrm{H})$; IR $(\mathrm{KBr}) v: 3410$, 3054, 2923, 2848, 1537, 1454, 1418, 1334, 1208, 1097, $1006,750 \mathrm{~cm}^{-1}$.

3,3'二(1-甲基吲哚基)苯基甲烷(3q): 得 $102 \mathrm{mg}$ 粉 红色固体, 产率 $73 \%$. m.p. $190 \sim 193{ }^{\circ} \mathrm{C}$ (lit. ${ }^{[19]} 185 \sim$ $\left.187{ }^{\circ} \mathrm{C}\right) ;{ }^{1} \mathrm{H}$ NMR $\left(400 \mathrm{MHz}, \mathrm{CDCl}_{3}\right) \delta: 7.37$ (dt, $J=7.9$, $1.0 \mathrm{~Hz}, 2 \mathrm{H}), 7.36 \sim 7.32(\mathrm{~m}, 1 \mathrm{H}), 7.29 \sim 7.24(\mathrm{~m}, 3 \mathrm{H})$, $7.22 \sim 7.16(\mathrm{~m}, 2 \mathrm{H}), 7.01 \sim 6.96(\mathrm{~m}, 2 \mathrm{H}), 6.52(\mathrm{~d}, J=0.8$ $\mathrm{Hz}, 2 \mathrm{H}), 5.87$ (s, 1H), 3.66 (s, 6H); IR (KBr) v: 3050, 3018, 2931, 1541, 1477, 1366, 1327, 1014, 741, $701 \mathrm{~cm}^{-1}$.

3,3'-二(1-甲基吲哚基)-(4-甲基苯基)甲烷(3r): 得 $106 \mathrm{mg}$ 粉红色固体, 产率 $73 \%$. m.p. $197 \sim 200{ }^{\circ} \mathrm{C}$ (lit. $\left.{ }^{[31]} 198 \sim 200{ }^{\circ} \mathrm{C}\right) ;{ }^{1} \mathrm{H}$ NMR $\left(400 \mathrm{MHz}, \mathrm{CDCl}_{3}\right) \delta: 7.38$ (dt, $J=8.0,0.9 \mathrm{~Hz}, 2 \mathrm{H}), 7.27$ (dt, $J=8.2,0.7 \mathrm{~Hz}, 2 \mathrm{H}$ ), $7.23 \sim 7.20(\mathrm{~m}, 2 \mathrm{H}), 7.20 \sim 7.15(\mathrm{~m}, 2 \mathrm{H}), 7.08(\mathrm{~s}, 1 \mathrm{H})$, $7.06(\mathrm{~s}, 1 \mathrm{H}), 7.00 \sim 6.95(\mathrm{~m}, 2 \mathrm{H}), 6.52(\mathrm{~d}, J=0.8 \mathrm{~Hz}, 2 \mathrm{H})$,
5.83 (s, 1H), 3.65 (s, 6H), 2.31 (s, 3H); IR (KBr) v: 3421, 3049, 2935, 1616, 1553, 1509, 1473, 1426, 1370, 1327, $1219,1149,1014,867,796,740 \mathrm{~cm}^{-1}$.

3,3'-二(2-甲基吲哚基)苯基甲烷(3s): 得 $133 \mathrm{mg}$ 粉 红色固体, 产率 95\%. m.p. 102 104 ${ }^{\circ} \mathrm{C}$ (lit. ${ }^{[2]} 247 \sim 248$ $\left.{ }^{\circ} \mathrm{C}\right) ;{ }^{1} \mathrm{H}$ NMR (400 MHz, $\left.\mathrm{CDCl}_{3}\right) \delta: 10.36(\mathrm{~s}, 2 \mathrm{H}), 7.47 \sim$ $7.43(\mathrm{~m}, 2 \mathrm{H}), 7.22 \sim 7.20(\mathrm{~m}, 5 \mathrm{H}), 6.91 \sim 6.87(\mathrm{~m}, 3 \mathrm{H})$, $6.85(\mathrm{~s}, 1 \mathrm{H}), 6.70 \sim 6.66(\mathrm{~m}, 2 \mathrm{H}), 5.93(\mathrm{~s}, 1 \mathrm{H}), 2.08(\mathrm{~s}$, $6 \mathrm{H}$ ); IR (KBr) v: 3386, 3055, 1456, 1299, 1018, $750 \mathrm{~cm}^{-1}$.

3,3'-二(2-甲基吲哚基)-(4-硝基苯基)甲烷(3t): 得 $153 \mathrm{mg}$ 棕色固体, 产率 97\%. m.p. 232 234 ${ }^{\circ} \mathrm{C}$ (lit. ${ }^{[2]}$ 239 241 ${ }^{\circ} \mathrm{C}$ ); ${ }^{1} \mathrm{H}$ NMR (400 MHz, $\mathrm{CDCl}_{3}$ ) $\delta: 10.87$ (s, $2 \mathrm{H}), 8.15(\mathrm{~d}, J=8.8 \mathrm{~Hz}, 2 \mathrm{H}), 7.42(\mathrm{~d}, J=8.8 \mathrm{~Hz}, 2 \mathrm{H}), 7.24$ (d, $J=8.0 \mathrm{~Hz}, 2 \mathrm{H}), 6.94 \sim 6.89(\mathrm{~m}, 2 \mathrm{H}), 6.81(\mathrm{~s}, 1 \mathrm{H}), 6.79$ (s, 1H), 6.76 6.68 (m, 2H), 6.07 (s, 1H), 2.10 (s, 6H); IR (KBr) $v: 3402,3386,3055,1600,1520,1485,1334,1109$, $1009,855,749 \mathrm{~cm}^{-1}$.

3,3'-二(2-甲基吲哚基)-(4-甲基苯基)甲烷(3u): 得 $137 \mathrm{mg}$ 棕色固体, 产率 94\%. m.p. 102 $104{ }^{\circ} \mathrm{C}$ (lit. ${ }^{[32]}$ $\left.106 \sim 108{ }^{\circ} \mathrm{C}\right) ;{ }^{1} \mathrm{H}$ NMR $\left(400 \mathrm{MHz}, \mathrm{CDCl}_{3}\right) \delta: 7.77$ (s, $2 \mathrm{H}), 7.21(\mathrm{~d}, J=8.0 \mathrm{~Hz}, 2 \mathrm{H}), 7.13(\mathrm{~d}, J=8.2 \mathrm{~Hz}, 2 \mathrm{H})$, $7.03 \sim 6.96(\mathrm{~m}, 6 \mathrm{H}), 6.85 \sim 6.80(\mathrm{~m}, 2 \mathrm{H}), 5.94(\mathrm{~s}, 1 \mathrm{H})$, 2.31 (s, 3H), 2.02 (s, 6H); IR (KBr) v: 3394, 3054, 2919, $1683,1619,1588,1508,1461,1299,1022,752 \mathrm{~cm}^{-1}$.

1,1'-(3,3'-二吲哚基-苯基)乙烷(3v): 得 $26 \mathrm{mg}$ 白色 固体, 产率 $19 \%$. m.p. $187 \sim 189{ }^{\circ} \mathrm{C}$ (lit. ${ }^{[30]} 189 \sim 190$ $\left.{ }^{\circ} \mathrm{C}\right) ;{ }^{1} \mathrm{H}$ NMR (400 MHz, $\left.\mathrm{CDCl}_{3}\right) \delta: 7.79$ (s, 2H), 7.40 $7.37(\mathrm{~m}, 2 \mathrm{H}), 7.34 \sim 7.32(\mathrm{~m}, 2 \mathrm{H}), 7.32 \sim 7.30(\mathrm{~m}, 2 \mathrm{H})$, $7.26 \sim 7.21(\mathrm{~m}, 2 \mathrm{H}), 7.21 \sim 7.17(\mathrm{~m}, 1 \mathrm{H}), 7.15 \sim 7.10(\mathrm{~m}$, $2 \mathrm{H}), 6.95 \sim 6.90(\mathrm{~m}, 2 \mathrm{H}), 6.59(\mathrm{~d}, J=2.4 \mathrm{~Hz}, 2 \mathrm{H}), 2.35(\mathrm{~s}$, 3H); IR (KBr) v: 3418, 3054, 2974, 2938, 1457, 1414, 1339, 1244, 1097, 1018, $737 \mathrm{~cm}^{-1}$.

1,1'-(3,3'-二吲哚基-甲基)乙烷(3w): 得 $90 \mathrm{mg}$ 白色 固体, 产率 $82 \%$. m.p. $158 \sim 160{ }^{\circ} \mathrm{C}$ (lit. ${ }^{[26]} 167 \sim 168$ $\left.{ }^{\circ} \mathrm{C}\right) ;{ }^{1} \mathrm{H}$ NMR $\left(400 \mathrm{MHz}, \mathrm{CDCl}_{3}\right) \delta: 7.80(\mathrm{~s}, 2 \mathrm{H}), 7.42 \sim$ $7.41(\mathrm{~m}, 1 \mathrm{H}), 7.40 \sim 7.39(\mathrm{~m}, 1 \mathrm{H}), 7.27(\mathrm{dt}, J=8.1,1.0 \mathrm{~Hz}$, $2 \mathrm{H}), 7.09 \sim 7.04(\mathrm{~m}, 2 \mathrm{H}), 6.99(\mathrm{~d}, J=2.4 \mathrm{~Hz}, 2 \mathrm{H}), 6.90 \sim$ 6.85 (m, 2H), 1.90 (s, 6H); IR (KBr) v: 3402, 3046, 2958, 1612, 1485, 1457, 1335, 1239, 1105, 1014, 819, $749 \mathrm{~cm}^{-1}$.

\section{References}

[1] (a) Porter, J. K.; Bacon, C. W.; Robins, J. D.; Himmel-sbach, D. S.; Higman, H. C. J. Agric. Food Chem. 1977, 25, 88.

(b) Osawa, T.; Namiki, M. Tetrahedron Lett. 1983, 24, 4719.

(c) Fahy, E.; Potts, B. C. M.; Faulkner, D. J.; Smith, K. J. Nat. Prod. 1991, 54, 564.

(d) Bell, R.; Carmell, N.; Sar. S. J. Nat. Prod. 1994, 57, 1587. 
(e) Garbe, T. R.; Kobayashi, M.; Shimizu, N.; Takesue, N.; Ozawa, M.; Yuka-wa, H. J. Nat. Prod. 2000, 63, 596.

[2] Rahimizadeh, M.; Eshghi, H.; Bakhtiarpoor, Z.; Pordel, M. J. Chem. Res. 2009, 269.

[3] Povszsz, L.; Katakin, G. P.; Foleat, S.; Malkovics, B. Acta Phys. Acad. Sci. Hung. 1996, 29, 299.

[4] Jitendra, R. S.; Kalpesh, D. P.; Radha, V. J. Catal. Commun. 2008, 9, 1071.

[5] Gong, H.-W.; Xie, Z.-F. Chin. J. Org. Chem. 2012, 32, 1195 (in Chinese). (宫海伟, 解正峰, 有机化学, 2012, 32, 1195.)

[6] Azizian, J.; Teimouri, F.; Mohammadizadeh, M. R. Catal. Commum. 2007, 8, 1117.

[7] Peng, Y. Y.; Zhang, Q. L.; Yuan, J. J.; Cheng, J. P. Chin. J. Chem. 2008, 26, 2228.

[8] Ghorbani-Vaghei, R.; Veisi, H.; Keypour, H. Mol. Diversity 2010, $14,87$.

[9] Yadav, J. S.; Gupta, M. K.; Jain, R.; Yadav, N. N.; Subba Reddy, B. V. Monatsh. Chem. 2010, 14, 1001.

[10] Li, J.-T.; Lin, Z.-P. Chin. J. Org. Chem. 2008, 28, 1238 (in Chinese)

(李记太, 萄志平, 有机化学, 2008, 28, 1238.)

[11] Pore, D. M.; Desai, U. V.; Thopate, T. S.; Wadgaonkar, P. P. ARKIVOC 2006, (xii), 75 .

[12] (a) Ji, S. J.; Wang, S. Y.; Zhang, Y.; Loh, T. P. Tetrahedron 2004, 60, 2051.

(b) Bandgar, B. P.; Shaikh, K. A. Tetrahedron Lett. 2003, 44, 1959.

[13] Yi, W.-G.; Jia, Z.-Y.; Li, N.-B.; Qiu, R.-H.; Chen, J.-Y.; Xu, X.-H. Chin. J. Org. Chem. 2012, 32, 2390 (in Chinese).

(易卫国，贾振永，李宁波，邱仁华，陈锦杨，许新华，有机化学， 2012, 32, 2390.)

[14] Wang, H.-S.; Zhao, L.-F. Chin. J. Org. Chem. 2005, 25, 869 (in
Chinese).

(王宏社, 赵立芳, 有机化学, 2005, 25, 869.)

[15] Chen, D.; Yu, L.; Wang, P. G. Tetrahedron Lett. 1996, 37, 4467;

[16] Nagarajan, R.; Perumal, P. T. Tetrahedron. 2002, 58, 1229.

[17] Babu, G.; Sridhar, N.; Perumal, P. T. Synth. Commun. 2000, 30, 1609.

[18] Mo, L. P.; Ma, Z. C.; Zhang, Z. H. Synth. Commun. 2005, 1997.

[19] Qu, H. E.; Xiao, C.; Hu, Q. S.; Wang, N.; Yu, K. H.; Liu, L. X. Molecules 2011, 16, 3855.

[20] Nagawade, R. R.; Shinde, D. B. Acta Chim. Slov. 2006, 53, 210.

[21] Terada, M.; Sorimachi, K.; Uraguchi, D. Synlett 2006, 0133.

[22] He, Q. L.; Sun, F. L.; Zheng, X. J.; You, S. L. Synlett 2009, 1111.

[23] Wang, X. B.; He. L.; Jian, T. Y.; Ye, S. Chin. Chem. Lett. 2012, 23, 13.

[24] Wang, X.-B.; Jian, T.-Y.; Ma, X.-W.; He. L. Chin. J. Org. Chem. 2012, 32, 2181 (in Chinese). (王湘波，简腾跃，马晓伟，何林，有机化学, 2012, 32, 2181.)

[25] Li, J. T.; Sun, S. F. Eur.-J. Chem. 2010, 7(3), 922.

[26] Ghorbani-Vaghei, R.; Veisi, H.; Keypour, H.; Dehghani- Firouzabadi, A. A. Mol. Diversity 2010, 14, 87.

[27] Vahdat, S. M.; Khaksar, S.; Baghery, S. Lett. Org. Chem. 2012, 9, 138.

[28] Nagawade, R. R.; Shinde, D. B. Bull. Korean Chem. Soc. 2005, 26, 1962.

[29] Ghorbani-Vaghei, R.; Malaekehpoor, S. M. Org. Prep. Proced. Int. 2010, 42,175

[30] Sheng, S. R.; Wang, Q. Y.; Ding, Y.; Liu, X. L.; Cai, M. Z. Catal. Lett. 2009, 128, 418.

[31] Zhang, Y.; Chen, X.; Liang, J.; Shang, Z.-C. Synth. Commun. 2011, 41, 2446.

[32] Rajitha, B.; Reddy, P. N.; Kumar, B. S.; Sreenivasulu, N.; Reddy, Y. R. T. J. Chem. Res. 2005, 4, 222.

(Li, L.; Lu, Z.) 